\title{
An empirical study of algorithms for point feature label placement
}

\section{Citation}

Stuart M. Shieber, Jon Christensen, and Joe Marks. An empirical study of algorithms for point feature label placement. Transactions on Graphics, 14(3), July 1995.

\section{Published Version}

http://dx.doi.org/10.1145/212332.212334

\section{Permanent link}

http://nrs.harvard.edu/urn-3:HUL.InstRepos:2032678

\section{Terms of Use}

This article was downloaded from Harvard University's DASH repository, and is made available under the terms and conditions applicable to Other Posted Material, as set forth at http:// nrs.harvard.edu/urn-3:HUL.InstRepos:dash.current.terms-of-use\#LAA

\section{Share Your Story}

The Harvard community has made this article openly available.

Please share how this access benefits you. Submit a story.

Accessibility 
[To appear in ACM Transactions on Graphics]

\title{
An Empirical Study of Algorithms for Point-Feature Label Placement
}

\author{
Jon Christensen \\ Harvard University \\ Cambridge, Massachusetts
}

\author{
Joe Marks \\ Mitsubishi Electric Research Laboratories \\ Cambridge, Massachusetts
}

\author{
Stuart Shieber \\ Harvard University \\ Cambridge, Massachusetts
}

\begin{abstract}
A major factor affecting the clarity of graphical displays that include text labels is the degree to which labels obscure display features (including other labels) as a result of spatial overlap. Point-feature label placement (PFLP) is the problem of placing text labels adjacent to point features on a map or diagram so as to maximize legibility. This problem occurs frequently in the production of many types of informational graphics, though it arises most often in automated cartography. In this paper we present a comprehensive treatment of the PFLP problem, viewed as a type of combinatorial optimization problem. Complexity analysis reveals that the basic PFLP problem and most interesting variants of it are NP-hard. These negative results help inform a survey of previously reported algorithms for PFLP; not surprisingly, all such algorithms either have exponential time complexity or are incomplete. To solve the PFLP problem in practice, then, we must rely on good heuristic methods. We propose two new methods, one based on a discrete form of gradient descent, the other on simulated annealing, and report on a series of empirical tests comparing these and the other known algorithms for the problem. Based on this study, the first to be conducted, we identify the best approaches as a function of available computation time.
\end{abstract}

CR Categories: H.5.2 [Information Interfaces and Presentation]: User Interfaces—screen design. 2.1 [Artificial Intelligence]: Applications and Expert Systems_cartography. I.3.5 [Computer Graphics]: Computational Geometry and Object Modeling_-geometric algorithms, languages, and systems.

General Terms: algorithms, experimentation.

Additional Key Words and Phrases: label placement, automated cartography, stochastic methods, simulated annealing, heuristic search.

\section{Introduction}

Tagging graphical objects with text labels is a fundamental task in the design of many types of informational graphics. This problem is seen in its most essential form in the field of cartography, where text labels must be placed on maps while avoiding overlaps with cartographic symbols and other labels, though it also arises frequently in the production of other graphics (e.g., scatterplots). Although several techniques have been reported for automating various label-placement tasks, the positioning of labels is still performed manually in many applications, even though it can be very tedious. ${ }^{1}$ Determining an optimal positioning of the labels is, consequently, an important problem.

In cartography, three different label-placement tasks are usually identified: labeling of area features (such as oceans or countries), line features (such as rivers or roads), and point features (such as cities or mountain peaks) (Imhof, 1962; 1975). While it is true that determining the optimal placement of a label for an isolated point feature is a very different task from

\footnotetext{
${ }^{1}$ Cook and Jones (1990) report that cartographers typically place labels at the rate of only 20 to 30 labels per hour, with map lettering contributing up to half of the time required for producing high-quality maps.
} 
determining the optimal placement of a label for an isolated line or area feature, the three placement tasks share a common combinatorial aspect when multiple features are present. The complexity arises because the placement of a label can have global consequences due to label-label overlaps. This combinatorial aspect of the label-placement task is independent of the nature of the features being labeled, and is the fundamental source of difficulty in automating label placement. We therefore concentrate on point-feature label placement (PFLP) without loss of generality; in Section 5 of the paper we describe how our results generalize to labeling tasks involving line and area features.

The PFLP problem can be thought of as a combinatorial optimization problem. Like all such problems, two aspects must be defined: a search space and an objective function.

Search space. An element of the search space can be thought of as a function from point features to label positions, which we will call a labeling. The set of potential label positions for each point feature therefore characterizes the PFLP search space. For most of the published algorithms, the potential label positions are taken, following cartographic standards, from an explicitly enumerated set. Figure 1 shows a typical set of eight possible label positions for a point feature. Each box corresponds to a region in which the label may be placed. Alternatively, a continuous placement model may be used, for example by specifying a circle around the point feature that the label must touch without intersecting.

In certain variants of the PFLP problem, we allow a labeling to omit certain points and their labels (presumably those that are most problematic to label, or least significant to the labeling application). When this option is included, the PFLP problem is said to include point selection. ${ }^{2}$

\begin{tabular}{|l|l|}
\hline 2 & 1 \\
\hline 3 & 4 \\
\hline
\end{tabular}

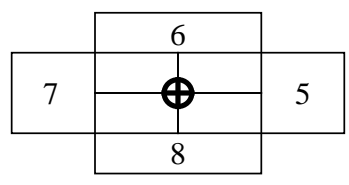

Figure 1: A set of potential label positions and their relative desirability. Lower values indicate more desirable positions.

Objective function. The function to be optimized, the objective function, should assign to each element of the search space (a potential labeling of the points) a value that corresponds to the relative quality of that labeling. The notion of labeling quality has been studied by cartographers, most notably by Imhof $(1962 ; 1975)$. However, Imhof's analysis is descriptive, not prescriptive; coming up with an appropriate definition of the objective function for a general label-placement problem (that is, one that includes point, line, and area features) is a difficult task. Labeling quality can depend on many factors, including detailed "world knowledge" and characteristics of human visual perception. Many of the label-placement algorithms reported in the literature therefore incorporate sophisticated objective functions. A popular approach has been to use a rule-based paradigm to encode the knowledge needed for the objective function (Ahn and Freeman, 1984; Freeman and Ahn, 1987; Jones, 1989; Cook and Jones, 1990; Doerschler and Freeman, 1992). For the PFLP problem, however, a relatively simple objective function suffices. Our formulation of the objective function is due to Yoeli (1972). ${ }^{3}$ In Yoeli's scheme, the quality of a labeling depends on the following factors:

- The amount of overlap between text labels and graphical features (including other text labels);

- A priori preferences among a canonical set of potential label positions (a standard ranking is shown in Figure 1); and

- The number of point features left unlabeled. (This criterion is pertinent only when point selection is incorporated into the PFLP problem.)

\footnotetext{
${ }^{2}$ In many types of production-quality maps, overplots are preferred to exercising point selection (Ebinger and Goulette, 1990).

${ }^{3} \mathrm{~A}$ recent study conducted by Wu and Buttenfield (1991) addresses the issue of placement preference for point-feature labels in more detail.
} 
Figure 2 illustrates these factors. By specifying how to compute a numerical score for each of the criteria above, an objective function can be defined. Such a function assigns to each labeling a number that indicates its relative quality. We will assume that low scores correspond to better labelings, so that the goal of the search is to minimize the objective function.

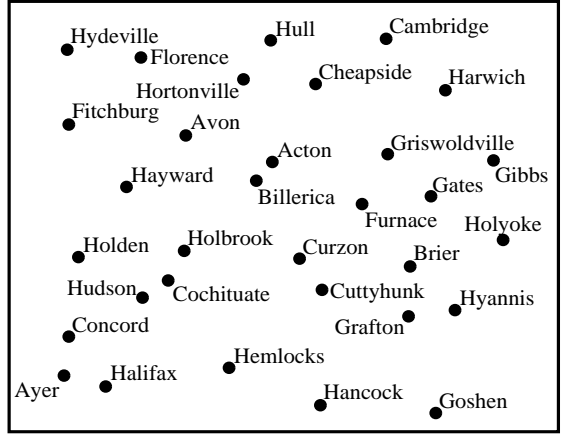

(a)

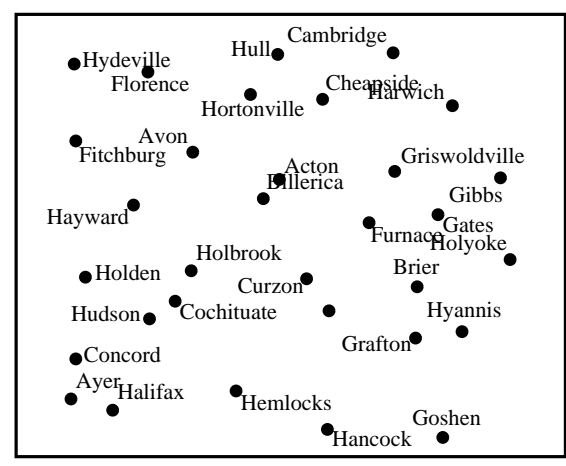

(b)

Figure 2: Good (a) and bad (b) labelings of the same map.

The PFLP problem is a combinatorial optimization problem defined by its search space and objective function; we wish to identify a general algorithm that is able to find a relatively good element of the search space. A natural issue to raise, before exploring possible search algorithms, is the intrinsic complexity of this search problem. In Section 2 we summarize some previous results that show that the problem and many of its interesting variants are NP-hard. Thus, any complete search algorithm will be impractical, any practical algorithm incomplete. ${ }^{4}$ This characterization is borne out by previously published algorithms, which fall into two classes: exhaustive search algorithms and local search algorithms. We review these algorithms in Section 3. As expected, the exhaustive algorithms are computationally profligate, and therefore impractical for realistically sized labeling instances.

We also present two new algorithms for the PFLP problem in Section 3. The first is a local search technique based on a discrete form of gradient descent. Although it is also incomplete, its performance on problems with high label density and its efficiency make it attractive under certain circumstances. The second technique is a stochastic algorithm based on simulated annealing. An extensive empirical comparison of all the algorithms, the first comparative study of label-placement heuristics, is presented in Section $4 ;{ }^{5}$ it illustrates the advantages of the new methods and provides recommendations for selecting a labeling algorithm.

\section{The Computational Complexity of PFLP}

In this section, we review some recent results on the inherent complexity of PFLP that have implications for algorithm design. To demonstrate the inherent complexity of the problem (and, subsequently, to compare various algorithms for the task), we must decide upon a particular instance of search space and objective function. We begin with a relatively simple version of the problem. Once this simplified problem is shown to be NP-hard, it is straightforward to demonstrate that more complicated variants of the problem are also NP-hard.

Our initial statement of the PFLP problem assumes a discrete placement model comprising four equally favored candidate positions, those numbered 1 through 4 in Figure 1. Point selection is not allowed and the objective function to be minimized is the number of point features labeled with one or more overplots. This simplified PFLP problem statement is an optimization problem. In order to apply the theory of NP-completeness to PFLP, we formulate a corresponding decision problem. For any given PFLP problem instance, we can ask the question: Is there an admissible labeling, a labeling with a score of zero, in

\footnotetext{
${ }^{4}$ This holds, of course, only if $\mathrm{P} \neq \mathrm{NP}$, as is commonly believed.

${ }^{5}$ Brief summaries of this work have appeared elsewhere (Christensen et al. 1993; 1994).
} 
which no labels overlap and no point features are obscured? The NP-completeness of this admissible-labeling problem has been established independently by at least three different teams of researchers (Kato and Imai, 1988; Marks and Shieber, 1991; Formann and Wagner, 1991). An algorithm for the PFLP optimization problem could always be used to solve the admissiblelabeling problem: find an optimal labeling and check to see whether the cost is 0 . Thus the PFLP optimization problem is at least as difficult as the admissible-labeling problem; in other words, the admissible-labeling result implies that optimal PFLP is NP-hard.

In spite of the apparent intractability of the basic problem, some simple restrictions can reduce the complexity dramatically. For example, a placement model that allows only two potential positions for each label results in a problem that is solved easily in polynomial time (Formann and Wagner, 1991). Similarly, the restricted set of problem instances in which no potential label position overlaps more than one other potential label position can also be solved efficiently. ${ }^{6}$ However, these polynomially solvable subcases notwithstanding, the previous complexity results imply that PFLP problems likely to be of practical interest are NP-hard.

If label sizes are held steady, increasing the scale of a map makes more room for labels. This observation leads to the following question: how much must the scale be increased to permit an admissible labeling for a given PFLP problem instance? Formann and Wagner have developed an efficient algorithm for this problem that is guaranteed to find an admissible labeling with a map scale no more than twice optimal (Formann and Wagner, 1991).

The recent complexity results make it clear that practical variants of PFLP, including all those discussed in this paper, are almost certainly intractable. Thus the failure of previous researchers to find an exact, polynomial-time algorithm for PFLP is not surprising-it is extremely unlikely that anyone will ever discover such an algorithm. Instead, research efforts should be directed towards powerful heuristic methods that may not exhibit guaranteed performance bounds, but that may work acceptably in practice. Several such algorithms are described and compared in the next section.

\section{Algorithms for PFLP}

Previously proposed PFLP algorithms fall into two main classes: those that perform a potentially exhaustive global search for an acceptable or optimal labeling, and those that perform search on a local basis only.

\subsection{Exhaustive search: naive or clever}

Exhaustive search algorithms for constraint satisfaction are often categorized based on the manner in which backtracking is performed. As an example, consider an algorithm that enumerates points in a prescribed order and places each label in a position that is currently unobstructed. If, as the algorithm proceeds, a point cannot be labeled (either because there are no positions without conflict, or because all available positions have been tried), the algorithm returns to the most recently labeled point and considers the next available position. The algorithm continues in this way until an acceptable labeling is identified or until the entire search space has been exhausted. A variety of modifications can be made to this algorithm in the hope of improving its performance. Heuristics include variable ordering, value ordering, returning to the source of failure, and various pruning techniques (Korf, 1988).

Exhaustive search algorithms like these have formed the basis for numerous reported algorithms for label placement (Ahn and Freeman, 1984; Freeman and Ahn, 1987; Noma, 1987; Freeman, 1988; Jones, 1989; Cook and Jones, 1990; Ebinger and Goulette, 1990; Doerschler and Freeman, 1992; Consorti et. al, 1993). While these algorithms perform acceptably for relatively small problems, in practice the exponential nature of the search space quickly overcomes the heuristics for even moderately sized problems, making the approach of exhaustive search impractical as a general solution to the PFLP problem, regardless of the sophistication of the particular heuristic. Indeed, the widespread use of exhaustive search techniques for the combinatorial aspects of the label-placement problem is something of a mystery. Zoraster (1991) notes that part of the problem might be the inappropriate use of expert-system technology: whereas a rule-based approach is useful in general label placement for determining potential label positions and for evaluating candidate labelings, it suggests, misleadingly, that rule-based techniques—exhaustive search is easy to implement in a rule-based system—are useful for all aspects of label placement.

\footnotetext{
${ }^{6}$ Developing an efficient algorithm for this artificial problem is left as an exercise for the interested reader.
} 


\subsection{Greedy algorithms}

A more practical approach to search results from avoiding the unbounded backtracking strategy of the exhaustive methods altogether. By limiting the scope of the search, more efficient algorithms can be devised. Of course, these algorithms may not find optimal solutions, but the hope is that a suitable trade-off between labeling quality and computational cost can be found.

Instead of undoing previously computed label placements, as with exhaustive search and its variants, any point whose label cannot be placed can be treated summarily: the point can be left out if point selection is allowed (Langran and Poiker, 1986), or it can be labeled even though a label overlap or feature obscuration results. (A third option, that of appealing to a human oracle for assistance, is noted by Yoeli (1972) as a practical alternative.) Such a "greedy algorithm" for PFLP yields behavior that is effective for a much more realistic space of problems, although the lack of backtracking certainly impairs the quality of the solutions that are found. For a greedy algorithm to be at all successful in identifying reasonable labelings, it is essential that heuristics for guiding the search, such as those mentioned in Section 3.1, be used. Even then, there is typically much improvement that can be made to the resulting labelings, as will be shown subsequently.

\subsection{Discrete gradient descent}

The quality of labelings produced by a greedy algorithm can be improved dramatically if the labelings are repaired subsequently by local alteration. This is the motivation for the gradient-descent algorithms presented below. A gradient-descent method is defined relative to a set of operations that specify ways in which one or more labels can be repositioned simultaneously. The basic idea of gradient descent is to choose from among the set of available operations the one that yields the most immediate improvement. By repeatedly applying the operation that most improves the labeling (or, equivalently, the operation that causes the most movement in the direction of the objective-function gradient), a new labeling can be computed that is significantly superior to the original. Again we present a straw man to exemplify the idea. Let the set of operations comprise those that move a single label arbitrarily from one potential position to another. An outline of the resulting algorithm, which we call discrete gradient descent is given below:

1. For each feature, place its label randomly in any of the available candidate positions.

2. Repeat until no further improvement is possible:

(a) For each feature, consider moving the label to each of the alternative positions.

(b) For each such repositioning, calculate the change in the objective function that would result if the label were moved.

(c) Implement the single label repositioning that results in the most improvement. (Ties are resolved randomly.)

In practice the algorithm precomputes a table of costs associated with each possible repositioning. After each label positioning, only elements of the table that touch the old or new label positions are recomputed.

\section{Local minima}

The major weakness of the discrete gradient-descent algorithm is its inability to escape from local minima of the objective function. Figure 3 shows a typical example of a local minimum. (The examples of local minima in this section, and those discussed for the Hirsch and Zoraster algorithms, though artificially constructed, are idealized versions of local minima that arose during experimentation with actual maps.) In this case, the conflict can be resolved by moving the lower feature's label to its bottom-left position and the upper feature's label to its upper-right position. Unfortunately, making any single move has no effect on the value of the objective function, and, because the algorithm only accepts changes that show an immediate improvement, the algorithm is unaware of the possibility of accepting a neutral move in order to make an improvement. Adjusting the algorithm to allow it to make moves that do not affect the objective function might remedy this particular example, but is not sufficient in general. In the example of Figure 4, the current value of the objective function could be improved from four obstructed labels (Figure 4a) to three (Figure 4b) by moving the four middle labels to their left-most positions. However any one of these moves will initially result in an uphill step and an intermediate score of 5. To limit the incidence of such local minima, more sophisticated gradient-descent heuristics have been devised. Nevertheless, as we will see, even the simplest discrete gradient-descent method performs surprisingly well. 


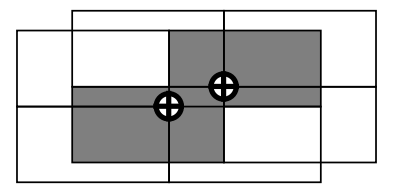

Figure 3: A local minimum of the discrete gradient-descent algorithm. The candidate label positions are marked with boxes, and the selected label positions are shaded.

(a)

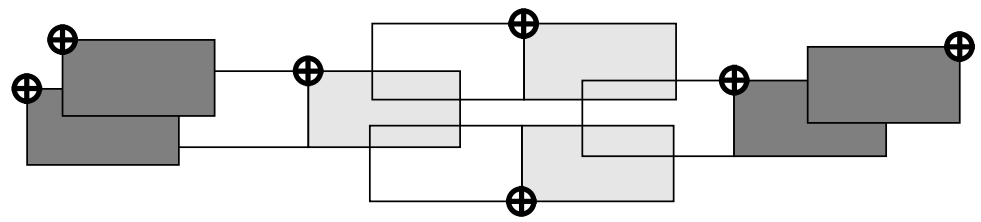

(b)

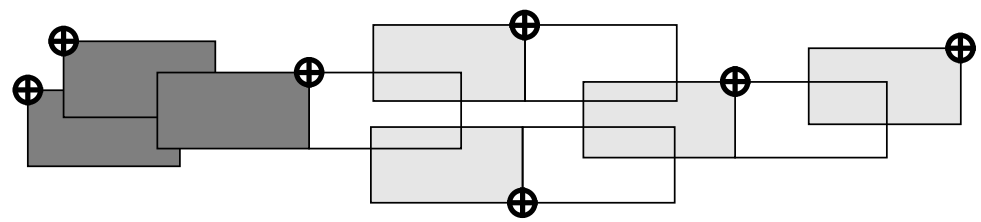

Figure 4: Another local minimum of the discrete gradient-descent algorithm (a) and an optimal configuration (b). The candidate label positions are marked with boxes, and selected label positions are shaded. Obstructed label positions are shaded dark.

\subsection{Approximating the gradient with overlap vectors}

Hirsch (1982) presents a more sophisticated gradient-descent method for PFLP. ${ }^{7.8}$ Hirsch's algorithm uses a continuous placement model in which each point feature has an infinite set of potential label positions. The potential positions for a point touch, but do not intersect, a circle centered about the point; labels are allowed to slide continuously around a circle (see Figure 5a). When the label touches at the highest, lowest, left-most, or right-most points of the circle, it is considered to be in a special zone and is allowed to slide back and forth along the point of tangency (see Figure 5b).

Initially each label is placed in the special zone to the right of its point. Each label is then tested for overlaps with other labels and intersections with the circular boundaries of other points. For each conflict an overlap vector is computed based on the $x$ and $y$ extents of the overlap or intersected area. Each overlap vector is split between the two conflicting features and represents the movement required to eliminate a particular conflict. The sum of overlap vectors associated with each label is then calculated to give an aggregate vector that represents (in an intuitive sense) a good direction in which to move the label so as to eliminate the overlaps and intersections. In Figure 5c the overlap vectors are drawn in light gray, and the aggregate vectors in black. (For labels involved in only one conflict the single overlap vector and the aggregate vector are the same.)

\footnotetext{
${ }^{7}$ A more elaborate version of this approach is described in U.S. Patent \#5,355,314 (Feigenbaum, 1994). The algorithm described therein differs from Hirsch's method in the following ways: in addition to repulsive forces from other labels and features, labels experience attractive forces from the cartographic features that they tag; labels initially start out very small and are grown to their full size over the course of the physical simulation; the coefficients in the various force formulae are set so that conflicts lead to a "marginally stable" system in which conflicted labels can be subjected to strong forces sufficient to escape some local minima; moving labels encounter frictional forces that help to dampen oscillations; and the Imhof standards for point-feature label preferences are incorporated through various algorithm-specific heuristics. This algorithm is currently in commercial use. Due to its recent publication, we were unable to include the algorithm in our comparative study.

${ }^{8}$ Mower (1986; 1993) describes an approach that shares characteristics of both Hirsch's algorithm (relaxation and constraint-propagation) and depth-first search (features are treated serially instead of in parallel as Hirsch does).
} 


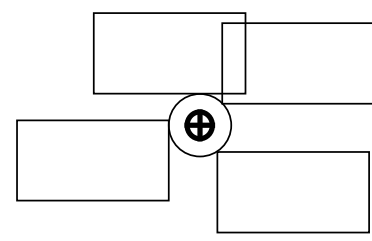

(a)

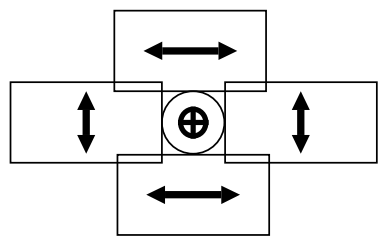

(b)

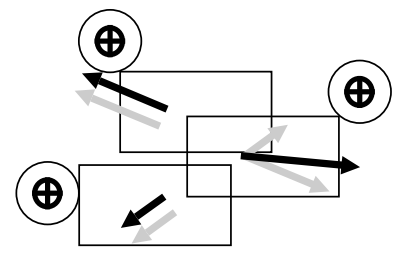

(c)

Figure 5: Some example potential label positions for Hirsch's algorithm (a), along with the special zones (b), and an example of overlap vectors (c).

Once an aggregate overlap vector has been calculated for each label, the algorithm seeks to move each label in the general direction of this vector in an effort to reduce the number of overlaps. The heuristic technique employed involves two styles of movement, an incremental movement around the circle and an absolute movement, which shifts the label directly to the point on the circle indicated by the overlap vector. Thus there are only two basic operations available for altering a labeling, but each operation is applied to all point features on a given round of application so that many labels may change positions simultaneously. The absolute movement repositions the label directly to the position indicated by the aggregate vector, regardless of the label's current position. The incremental movement, on the other hand, involves a series of heuristic rules that move the label in the direction of the aggregate overlap vector. Hirsch suggests alternating between the two movement styles, with more frequent application of the incremental movement.

The intuition behind the algorithm is best explained by an analogy with a physical system. The individual overlap vectors represent a "force" of repulsion between overlapping objects, the sum an aggregate force. Thus, through gradual movements, the system settles into a local minimum of the "energy" of the system. The overlap vectors approximate the gradient in the energy space. To allow some ability to exit from local minima, the absolute movements are designed to allow a jump from one energy state to another, hopefully lower one.

There are two sources of problems for Hirsch's algorithm. First, since the overlap vectors provide only an approximation of the gradient, they are subject to error. Second, like the discrete gradient-descent algorithm, Hirsch's algorithm is susceptible to getting stuck in local minima.

\section{Gradient approximation errors}

A typical dilemma is due to the summation of overlap vectors. When multiple labels overplot a single label, the magnitude of the calculated aggregate vector will often be unnecessarily large, leading to problems of overshooting during incremental movements.

Note also that Hirsch's overlap vectors each exhibit two degrees of freedom, whereas the labels are constrained to lie tangent to their associated circles. The result is that even in those cases where the accumulated overlap vector represents a favorable direction of movement, the particular manner in which a label is repositioned is often quite fragile. If a large component of the overlap vector points radially outward, for example, the location of the repositioned label is somewhat arbitrary.

\section{Local minima}

Hirsch's algorithm, like the discrete gradient-descent algorithm, can also get stuck in local minima. The nature of these minima is closely related to the specific heuristics the algorithm employs in response to various overlap situations. Figure 6 shows a problematic configuration. During applications of the incremental movement, the label is adjusted slightly up and down until it is centered between, but still conflicting with, the two labels above and below. During applications of the absolute-style movement, the horizontal component of the overlap vector dominates, and the label cycles between the left and right placements, missing the acceptable positions above and below the feature.

\section{Compensating for the placement model}

In order to compare the performance of Hirsch's algorithm against other PFLP algorithms, several issues relating to the placement model need to be addressed. The presence of a circular buffer surrounding each point feature handicaps the algorithm, disallowing free space that other algorithms are able to exploit, and forcing labels outward, thus increasing their effec- 


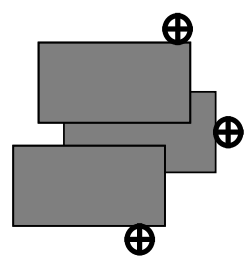

(a)

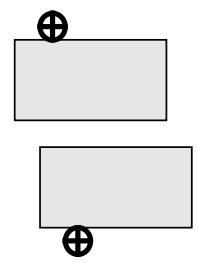

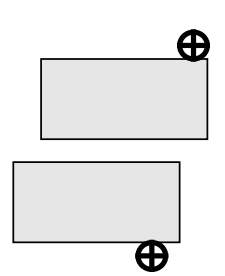

$\oplus$

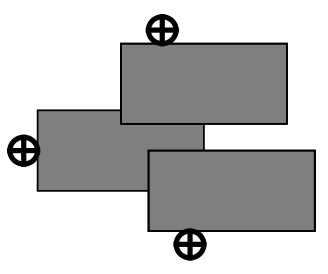

(b)

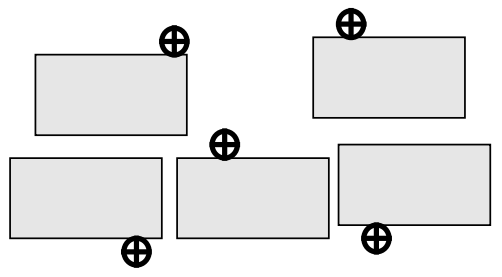

(c)

Figure 6: A local minimum of Hirsch's algorithm. The algorithm oscillates between configurations (a) and (b), unable to discover the preferred configuration (c).

tive dimensions. We considered two methods to compensate for this. First, we experimented with adjusting the label sizes for Hirsch's algorithm. We decreased the dimensions of each label such that the combined area of the placement circle and reduced label was equivalent to the area of the unmodified label. Second, we simply set the radius of the placement circle to zero. We found the latter method to perform slightly better on average, and included this variant of the algorithm in our comparisons. ${ }^{9}$ A related issue involves the continuous nature of the placement model. Since this allows a larger and therefore lessconstrained search space, this probably gives Hirsch's algorithm an advantage. Although this discrepancy is harder to resolve, a fairer comparison could be obtained by running the discrete algorithms with a 16- or 20-position placement model, as opposed to the four-position model used in the experiments. However, the results described in Section 4 render this point irrelevant.

\subsection{Mathematical programming for PFLP}

Next, we turn to an algorithm introduced by Zoraster $(1986 ; 1990)$ that addresses the optimization nature of PFLP directly by applying mathematical programming techniques to its solution. ${ }^{10}$ Zoraster begins by formulating PFLP as a $0-1$ integer programming (ZOLP) problem: ${ }^{11}$

- Given $K$ labels and $N_{k}$ possible positions for each label, each potential label position is represented by a variable $X_{i, k}, 1 \leq i \leq N_{k}$, and $1 \leq k \leq K$. (Point selection is achieved by specifying a special label "position" that indicates a deselected point.)

- Each $X_{i, k}$ has value 0 or 1 , indicating the absence or presence, respectively, of a label in that position.

- One set of constraints expresses the requirement that each point be labeled exactly once: $\sum_{i=1}^{N_{k}} X_{i, k}=1$ for $1 \leq k \leq K$.

\footnotetext{
${ }^{9}$ This is perhaps not surprising given the algorithm's predilection for label placements within special zones. Incremental movements tend to relocate labels into special zones, whereas only the absolute-style movements are able to move a label out of a special zone. Since the algorithm finishes with a series of 15 incremental movements, in practice nearly all labels finish in special zones.

${ }^{10}$ This algorithm is in commercial use in the oil industry to label drilling maps (Zoraster, 1990).

${ }^{11}$ Cromley (1986) has experimented independently with a slightly different ZOLP formulation of the label-placement problem.
} 
- Given $Q$ pairwise overlaps between possible label positions, a second set of constraints expresses the requirement that no two labels overlap: $X_{r_{q}, s_{q}}+X_{r_{q}^{\prime} s_{q}^{\prime}} \leq 1$ for each potential overlap, $1 \leq q \leq Q$.

- The objective function is $\sum_{k=1}^{K} \sum_{i=1}^{N_{k}} W_{i, k} X_{i, k}$, where $W_{i, k}$ is a weighting that represents placement preferences.

Because ZOLP is itself NP-hard (Sahni, 1974), a complete, efficient algorithm for the PFLP problem recast in this way is still not possible, but heuristic techniques for ZOLP can now be applied to the PFLP problem. Zoraster combines Lagrangian relaxation, subgradient optimization, and several problem-specific heuristics in his solution. The primary insight of Zoraster's algorithm is to relax the overplot constraints and include them as additional penalty terms in the objective function. This gives:

- Minimize $\sum_{k=1}^{K} \sum_{i=1}^{N_{k}} W_{i, k} X_{i, k}+\sum_{q=1}^{Q}\left(X_{r_{q} s_{q}}+X_{r_{q}^{\prime} s^{\prime} s_{q}}-1\right) d_{q}$

- Still subject to $\sum_{i=1}^{N_{k}} X_{i, k}=1$ for $1 \leq k \leq K$

In this modified objective function, the $d_{q} \geq 0$ are Lagrangian multipliers, one for each pairwise overplot constraint. Note that for a given set of Lagrangian multipliers, the minimum value of the objective function is easily identified by choosing the label-position variable with the smallest objective-function coefficient for each point feature. Although Lagrangian methods for ZOLP can be arbitrarily sophisticated, Zoraster's basic algorithm is a straightforward implementation of standard techniques (Fisher, 1981):

1. Compute and store the objective-function coefficient for each potential label position.

2. Generate a current labeling (CL) by picking the label position with the lowest objective-function coefficient for each point feature.

3. Initialize the active constraint set (ACS) to the empty set.

4. Repeat for 40 iterations or until a solution with no label conflicts is found:

(a) Identify all pairwise constraints that CL violates and add any new ones to ACS. (The Lagrangian multiplier of each newly introduced constraint is zero initially, so adding a new constraint to ACS does not affect the objective-function coefficients.)

(b) Make a local copy, CL', of CL.

(c) Repeat for $x$ iterations, where $x$ is the lower of 400 or the number of iterations required to find a feasible solution with respect to the current ACS, plus an additional 100 iterations if a feasible solution is found in the first 400 iterations: $^{12}$

i. Update CL' by picking the label position with the lowest objective-function coefficient for each point feature.

ii. Copy CL' to CL if it is better.

iii. If a constraint in ACS is overconstrained (i.e., both conflicting label positions are occupied), the corresponding Lagrangian multiplier is increased, thus increasing the objective-function coefficients for the two label positions involved.

iv. If a constraint in ACS is underconstrained (i.e., both conflicting label positions are not occupied), the corresponding Lagrangian multiplier is decreased, thus decreasing the objective-function coefficients for the two label positions involved.

\section{Return CL.}

\footnotetext{
${ }^{12}$ This inner loop constitutes the Lagrangian heuristic, with steps (iii) and (iv) constituting the subgradient optimization. Note that the Lagrangian heuristic will be solving relatively simplified versions of the full problem initially, because very few constraints will be included in ACS at first.
} 


\section{Local minima}

If the algorithm were implemented exactly as described above, it would perform quite poorly. The algorithm exhibits two weaknesses: a pronounced sensitivity to local minima, and a tendency to fall into useless cyclic behavior.

To address the worst of these deficiencies, Zoraster recommends a series of modifications to the basic algorithm. The first modification he suggests is rescaling the size of the multiplier increments used in 4(c)iii and 4(c)iv. If a specified number of iterations have passed without improving the best solution seen, the algorithm is assumed to be in a region surrounding a local minimum of the objective function. By reducing the multiplier increments periodically, the algorithm is often able to identify improved minima.

Even with this modification, the algorithm tends to cycle about local minima, continuously re-evaluating a particular sequence of labelings. If two features have overlapping label positions, for example, and both are currently occupied, then the associated objective-function coefficients of both positions will be increased. This will make them less attractive over time and it is likely that both labels will be simultaneously moved to alternate positions. On subsequent iterations, both positions will still overlap but are now unoccupied so their associated coefficients will decrease. This will make both positions relatively more attractive to their respective features and it often occurs that they will be simultaneously reoccupied. This situation is illustrated in Figure 7. In order to avoid this particular type of cyclic behavior, Zoraster discriminates in the overconstrained case, applying the multiplier to only one of the objective-function coefficients; the choice between coefficients is made by examining whether the algorithm is currently in an odd- or even-numbered iteration. This second modification proves to be crucial to the success of the algorithm though it has no motivation or analogue in the mathematical formulation.

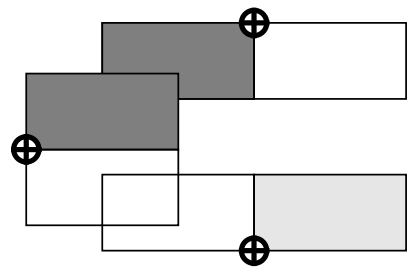

(a)

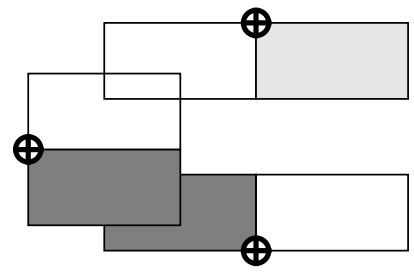

(b)

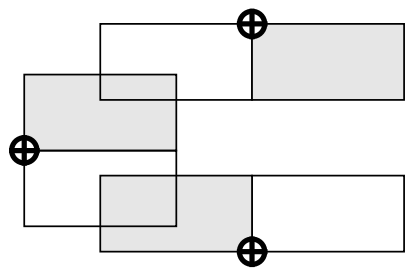

(c)

Figure 7: Stable and unstable configurations for Zoraster's approach. The conflict in configuration (a) causes the filled regions of the upper and left points to be disfavored, and the slack in the potential conflict between the lower and left points causes the unfilled regions for those two points to be favored. This leads eventually to modifying the configuration as in (b). This configuration, similarly, eventually leads back to the configuration in (a). The stable configuration (c) is never found.

A more insidious form of cycling can be caused by the intersection of more than two potential label positions. Overplots will gradually be discouraged, yet resolved overplots will result in underconstrained pairwise constraints, which in turn encourage surrounding labels to repopulate the contentious region. This situation is illustrated in Figure 8. Since the center candidate position overplot represents an underconstrained constraint, the left and right labels will be encouraged to move into the conflicted area, despite the fact that this will always introduce a conflict with the top label. As the number of label positions that overlap increases beyond three, the problem is exacerbated since label positionings are encouraged in regions that are often already dense with overplots. Zoraster attempts to address this deficiency by a third modification: arbitrarily pinning variables (i.e., fixing their values permanently) that are subject to four or more pairwise overplot constraints. If no feasible solution has been identified after 400 iterations of the Lagrangian heuristic, variables that are subject to more than three overplot constraints are pinned to zero. If after 600 iterations a feasible solution has still not been identified, the current (infeasible) solution is returned to the top level of the algorithm. This is equivalent to arbitrarily eliminating label positions in crowded areas of the map.

A fourth modification that attempts to control the algorithm's susceptibility to this weakness is the choice of multiplier increments. Zoraster recommends an initial overconstrained stepsize of $+\frac{1}{8}$ and an underconstrained stepsize of $-\frac{1}{16}$. The relative magnitudes of the stepsizes loosely represent the ability of a violated constraint to discourage subsequent reoccupation of a conflicted label position. Although Zoraster offers these values as empirical constants based on his experiments with a vari- 


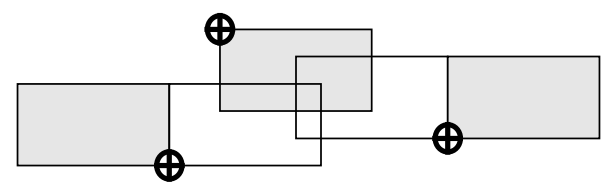

Figure 8: An unstable configuration for Zoraster's algorithm.

ety of different maps, optimal values are probably dependent on the density of the particular labeling problem. Indeed, we obtained better performance by using slightly modified parameter values and by making other subtle changes to the algorithm, as discussed elsewhere (Christensen et al., 1992).

\subsection{Stochastic search}

As we have seen, each of the local search methods can be trapped in local minima of the search space; the inherent intractability of the problem makes this inevitable for any practical algorithm. Nonetheless, we may still hope to improve upon the level of performance exhibited by these algorithms by examining more carefully the frailties that they exhibit.

The problems with the local search methods fall into two classes. First, there are systematic patterns on which the various algorithms get into trouble by getting trapped in local minima. As the number and density of points increases, the odds of seeing these patterns increase correspondingly, and performance may degrade. Second, the particular operations that the algorithms incorporate do not allow for jumping out of a local minimum once one is found. These two behaviors of systematicity and monotonicity are symptomatic of problems for which stochastic methods tend to work well. Stochastic methods, such as simulated annealing (Kirkpatrick, Gelatt Jr., and Vecchi, 1983; Cerny, 1985) and genetic algorithms (Holland, 1975), attempt to resolve the problems of systematicity and monotonicity by incorporating a probabilistic or stochastic element into the search. Since the stochastic course of behavior is unpredictable, systematic artifacts of the algorithm can be eliminated, and allowance can be made for a suitably limited, nonmonotonic ability to jump out of local minima. It seems natural then to apply a stochastic method to the PFLP problem.

\section{Simulated annealing for PFLP}

Simulated annealing (Kirkpatrick, Gelatt Jr., and Vecchi, 1983; Črny, 1985) is essentially a stochastic gradient-descent method that allows movement in directions other than that of the gradient. In fact, the solution is sometimes allowed to get worse rather than better. Of course, such anarchic behavior is not tolerated uniformly. Rather, the ability of the algorithm to degrade the solution is controlled by a parameter $T$, called the temperature, that decreases over time according to an annealing schedule. At zero temperature, such negative steps are disallowed completely, so that the algorithm reduces to a descent method (though not necessarily along the gradient). At higher temperatures, however, a wider range of the space can be explored, so that regions surrounding better local minima (and perhaps even the global minimum) may be visited. The following outline describes the essential characteristics of a simulated-annealing algorithm for PFLP:

1. For each point feature, place its label randomly in any of the available potential positions.

2. Repeat until the rate of improvement falls below a given threshold:

(a) Decrease the temperature, $T$, according to the annealing schedule.

(b) Pick a label and move it to a new position.

(c) Compute $\Delta E$, the change in the objective function caused by repositioning the label.

(d) If the new labeling is worse, undo the label repositioning with probability $P=1.0-e^{-\Delta E / T}$.

The implementation of a standard simulated-annealing algorithm involves four components: choice of an initial configuration, an appropriate objective function, a method for generating configuration changes, and an annealing schedule. 
Initial configuration. As an alternative to starting with randomly placed labels, one could consider a "piggyback" method where simulated annealing is applied as a post-process to the results of another algorithm. In our experiments, however, this did not lead to either a significantly better solution or faster convergence.

Objective function. The choice of objective function affects the aesthetics of the layout, the quality of the solution, and efficiency of the search. Because simulated annealing is a statistical method that relies on a large number of evaluations for its success, the best objective functions are those for which $\Delta E$ can be computed quickly. The objective functions we chose counted the number of obstructed labels (if point selection was disallowed) or the number of deleted labels plus the number of obstructed labels. If point selection is allowed, we also considered an objective function that counts the number of pairwise overplots plus the number of deleted labels. This change in objective function does not noticeably change the performance of the annealing algorithm, but has the advantage of being significantly faster to compute.

Configuration changes. We have experimented with two strategies for choosing which label to reposition: the label can be chosen randomly from the set of all labels, or it can be chosen randomly from the set of labels that are currently experiencing a conflict. The second method isolates changes to those parts of the map that have conflicts, causing the algorithm to converge faster. When cartographic preferences that distinguish label positions are included in the problem, this simplification is no longer acceptable because the movement of unconflicted labels may affect the current value of the objective function. In the experiments reported here, the more time-consuming method of choosing from all available features was used.

Annealing schedule. The initial value of $T$ was selected so that $P=\frac{2}{3}$ when $\Delta E=1$. At each temperature a maximum of $20 n$ labels are repositioned, where $n$ is the number of point features. The temperature is then decreased by 10 percent. We employ a Metropolis-style algorithm, always accepting a suggested configuration change if it leads to a lower cost. If more than $5 n$ successful configuration changes are made at any temperature, the temperature is immediately decreased. This process is repeated for at most 50 temperature stages. However, if the algorithm stays at a particular temperature for the full $20 \mathrm{n}$ steps without accepting a single label repositioning, then it stops with the current labeling as the final solution. We found the particular choice of annealing schedule to have a relatively minor affect on the performance of the algorithm as discussed in Section 4. This particular schedule was chosen to provide a reasonable trade-off between efficiency and solution quality; longer annealing schedules result in slightly improved solutions.

\section{Comparison Experiments}

In order to compare the effectiveness of this wide variety of algorithms for PFLP, we implemented six algorithms chosen from the set of non-exhaustive methods for PFLP. (Our experiments have shown that exhaustive methods are impractical for maps with as few as 50 point features.) The algorithms evaluated included a straw-man random-placement algorithm, in which label positions are assigned in a completely random fashion. This algorithm serves as an effective lower bound on algorithm performance. A greedy algorithm that serves as an efficient variant of the exhaustive methods described in Section 3.1 was also tested. The discrete gradient-descent algorithm was implemented, in addition to the algorithms of Hirsch and Zoraster. Finally, a stochastic algorithm utilizing simulated annealing was implemented. Each of the algorithms (except for Hirsch's) was allowed four candidate placement positions for labels. All candidate positions were taken to be equally desirable, i.e., preferences among different potential label positions were not considered (except where otherwise noted). A complete discussion of the implementation details for all of the algorithms is provided elsewhere (Christensen et al., 1992).

We began our comparison by testing the performance of each of the algorithms on randomly generated data, with and without point selection, to establish an overall ranking. To determine whether the relative performance of the algorithms was affected by the particular distribution, we then conducted similar tests on naturally occurring point-feature data. Next we ran a series of experiments on two gradient-descent variants in an attempt to improve on the best-seen solutions. Finally we investigated the effects of varying the annealing schedule of the simulated-annealing algorithm, and noted that the presence of cartographic preferences for candidate positions plays an important role in the usefulness of varying the annealing schedule. For this we conducted four additional trials, comparing the performance of three different annealing schedules while varying the use of point selection as well as the inclusion of cartographic preferences. 

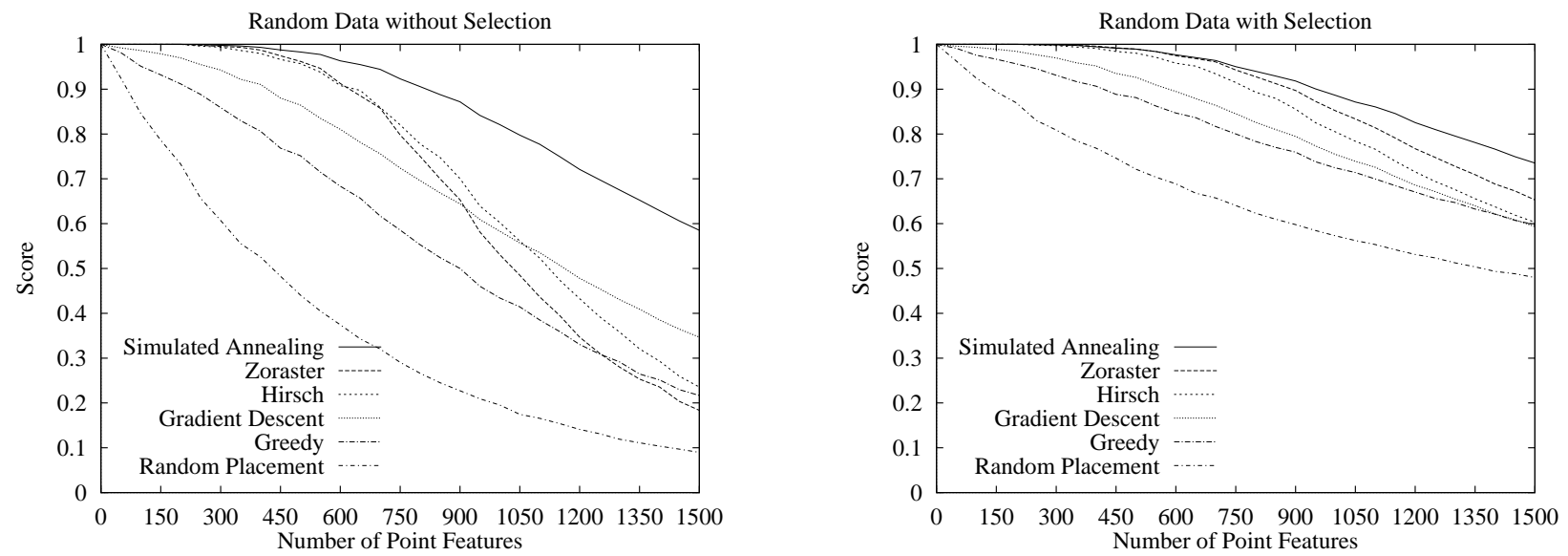

Figure 9: Results of empirical testing of six PFLP algorithms on randomly generated map data with point selection prohibited and allowed. The vertical axis shows the fraction of labels plotted without obstruction.

In the first group of tests, $n$ point features with fixed-size labels ( $30 \times 7$ units) were randomly placed on a region of size 792 by 612 . (These dimensions were selected subjectively in an effort to identify a typical map scale for an 11 by 8.5 inch page size.) Tests were run for $n=50,100,150, \ldots, 1500$. For each problem size tested, 25 layouts were generated, a score was calculated equal to the fraction of labels placed without overplots, and the results were averaged to give a composite result for the algorithm at that problem size. No penalty was assessed for label positions that extended beyond the boundary of the region. These tests were then repeated with point selection allowed. For most of the algorithms (greedy, gradient descent, Zoraster, and simulated annealing) this was a natural extension. For the Hirsch algorithm, however, there was no straightforward method of allowing points to be deleted. In order to include Hirsch's algorithm in the point-selection comparisons, we developed a post-pass deletion heuristic, which seeks to clear the map of overplots with the fewest number of label deletions possible. This heuristic deletes the feature whose label has the greatest number of conflicts with other (non-deleted) labels. This process is repeated until the map is free from overplots. Although this algorithm is clearly non-optimal (it is straightforward to show that optimal PFLP is reducible to the problem of optimal label deletion, which is therefore NP-hard), we found it to be an acceptable heuristic in practice. The score was again the fraction of labels placed without conflict. Figure 9 shows the results of these experiments. As these graphs show, simulated annealing performs significantly better across the full range of problems considered. Other perspectives on these results are shown in Figures 10 and 11. Figure 10 shows a particular random map of 750 point features labeled by the six basic algorithms. Figure 11 illustrates the variance across 25 different problem instances, for maps involving 750 and 1500 point features.

Next, cartographic data for Massachusetts were used to test the algorithms on naturally occurring point-feature distributions obtained from the GNIS state file for Massachusetts (United States Geological Survey 1990). The algorithms were again scored based on the number of unconflicted labels, both with and without point selection. At each problem size, 25 layouts were generated by choosing randomly from the data file. For example at $n=350$, each problem instance was generated by choosing 350 point features randomly from the GNIS data. Tests were run for $n=50,100,150, \ldots, 500$. Figure 12 shows the results of these tests. Because the ratio of average label size to available map area is significantly larger for the Massachusetts examples, and also due to clustering of the point features, the performance of the algorithms deteriorates faster in the experiments involving Massachusetts data (Figure 12) as compared with the randomly generated data (Figure 9). Nonetheless, the overall rankings are preserved.

Though the simulated-annealing algorithm easily dominated the competing algorithms, we noted that the discrete gradient-descent algorithm performed surprisingly well given its simplicity, especially at high densities, where it outperforms all methods but simulated annealing. To investigate this approach in more detail, we implemented two related algorithms, "2-opt" and "3-opt" discrete gradient-descent algorithms, which consider the best sequence of two and three repositionings at each iteration. ${ }^{13}$ A practical implementation of these algorithms is moderately complicated and requires a careful strategy for selective rescoring of repositionings at each iteration, supporting data structures for efficient search of a table of repositionings, and some clever record-keeping measures. Figure 13 shows the results of these new variants compared with the original discrete 
gradient-descent algorithm, the simulated-annealing algorithm and the random-placement algorithm. Although the "2-opt" and "3-opt" algorithms each improve on the performance of their predecessor, the degree of improvement grows less in each case, hinting towards an asymptote around the performance of the simulated-annealing algorithm. Further, even with a very careful implementation, the computational requirements of the 2-opt and 3-opt algorithms quickly become unreasonable as the number of candidate positions increases.

The next set of experiments investigated the effect of the annealing schedule on the performance of the simulated-annealing algorithm. We found that for very simple objective functions, e.g., the original four-position model without placement preferences, most potential label repositionings have no effect on the value of the objective function. For such spaces, a simple random descent (the equivalent of zero-temperature simulated annealing) performs nearly as well as simulated annealing at medium and even long schedules. This is seen in Figure 14. As the terrain of the search space becomes rougher, and involves a greater number of local minima, the utility of the annealing schedule is increased. Figure 15 shows that in experiments involving a four-position model with placement preferences, the performance of zero-temperature annealing drops roughly to that of the discrete gradient-descent algorithm. ${ }^{14}$

Computational resources required for the various algorithms vary dramatically, but not unexpectedly. As a rough indication of algorithm performance, Figure 16 depicts a scatterplot of running times for each of the algorithms running on a DEC 3000/400 AXP workstation. To the extent that these running times are representative of the intrinsic computational requirements of each algorithm, certain subsumption relationships can be derived. For example, Zoraster's algorithm lies to the lower right of the 3-opt discrete gradient-descent algorithm, indicating that it is both slower and exhibits inferior solutions. The 3-opt algorithm, in turn, is dominated by the simulated-annealing algorithm. Eliminating algorithms that are subsumed by other algorithms leaves a "staircase" of algorithms that, depending on requirements of time versus solution quality, would be preferred for a given task. At both densities shown, this staircase includes, in order of increased computation time and solution quality: random placement, the greedy algorithm, the original gradient-descent algorithm, Hirsch's algorithm at low densities, the 2-opt gradient-descent algorithm, and the simulated-annealing algorithm.

\section{Conclusions}

The point-feature label-placement problem is a graphics-design problem of practical importance and noted difficulty. Analysis of the computational complexity of the problem bears out its inherent difficulty; the search for good heuristic solutions thus becomes important. In this paper, we have proposed two new algorithms for PFLP-variants of discrete gradient descent and simulated annealing — and compared them with previously proposed algorithms. This empirical testing, which constitutes the first such comparative study, provides the basis for a graphic comparison of the time-quality tradeoff in labelplacement algorithms, demonstrating that certain algorithms-3-opt gradient descent, Zoraster's, and Hirsch's algorithm, for instance - are subsumed by others in both speed and quality. The experiments also argue for the use of simulated annealing over the alternatives when solution quality is critical. For time-critical applications, the annealing schedule can often be shortened or eliminated altogether while still providing reasonable solutions. This result stands in contrast to previous empirical investigations of simulated annealing, which have shown that for a few NP-hard problems simulated annealing is competitive with customized heuristic techniques, but typically only when allowed to run for very long periods of time (Johnson et al., 1989; 1991). Simulated annealing has the additional advantage of being one of the easiest algorithms to implement. Table 1 gives the number of lines of code for each of the algorithms under our implementation, as an admittedly rough indication of implementation complexity. ${ }^{15}$

\footnotetext{
${ }^{13}$ We use these terms because of the similarity of these methods to the $k$-opt methods proposed for the NP-complete Traveling Salesman Problem (TSP). Variants of this method comprise the current best algorithms for the TSP (Johnson, 1990).

${ }^{14}$ Note that the performance of the gradient-descent algorithm appears to have increased relative to the original experiments. Because the original objective function yields a search space with many flat plateaus, the algorithm is often unable to find the edge of a plateau and terminates; the modified objective function yields virtually no plateaus and the algorithm is able to continue further before reaching a local minimum. A second reason for the improvement is the inclusion of preferences in the score metric. Since the score considers a larger dynamic range, the scale of the graph along the vertical axis is more compressed, resulting in a closer grouping of the algorithms. (Notice the relatively higher performance of random placement as compared with the previous trials.)

${ }^{15}$ Our implementation makes extensive use of function pointers to provide dynamic reconfiguration of the basic aspects of each algorithm. As a result, however, these numbers are undoubtedly higher than those that would occur in more straightforward implementations.
} 


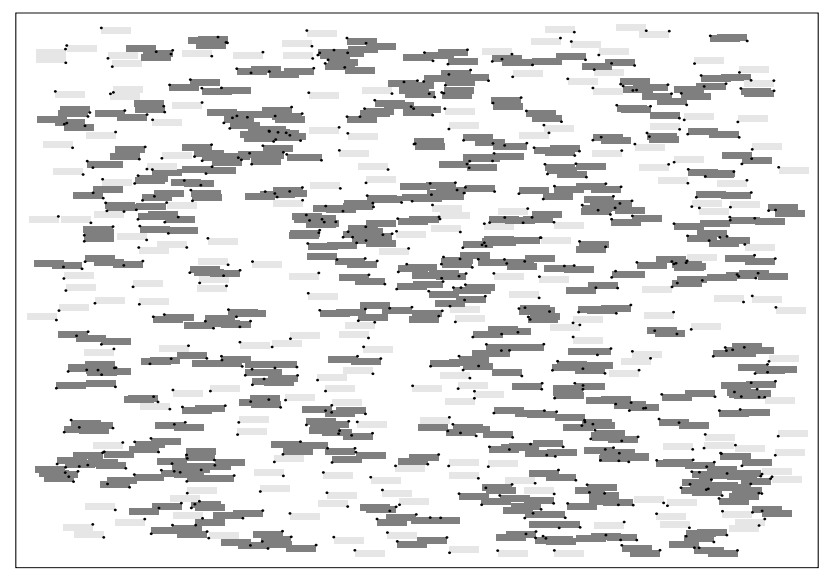

Random Placement (564)

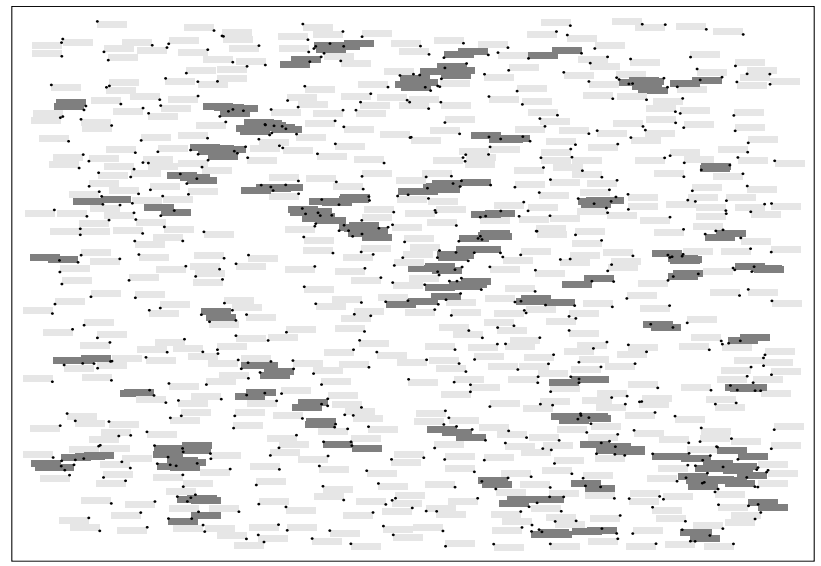

Discrete Gradient Descent (222)

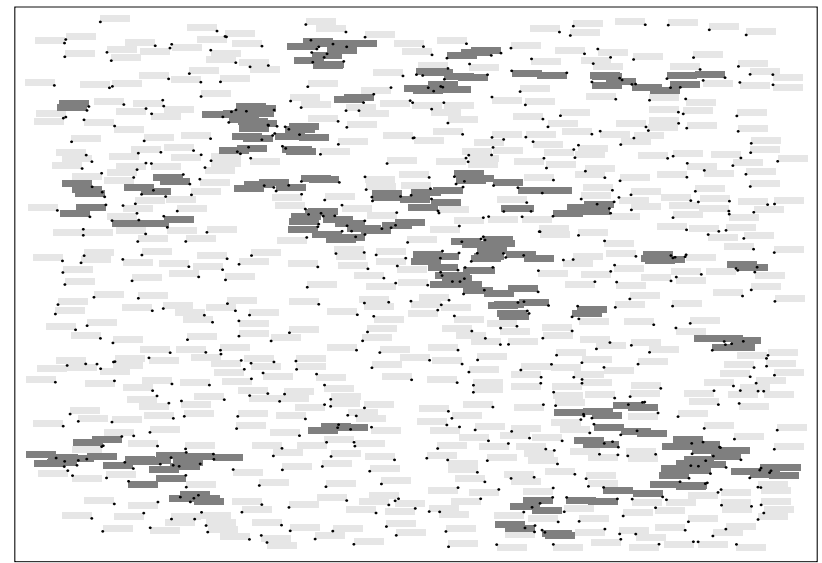

Zoraster's Algorithm (219)

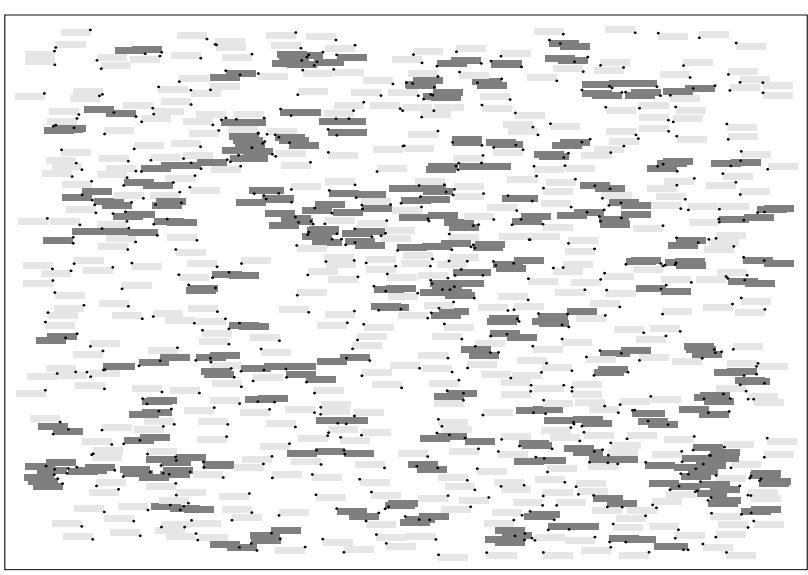

Greedy Depth-First Placement (341)

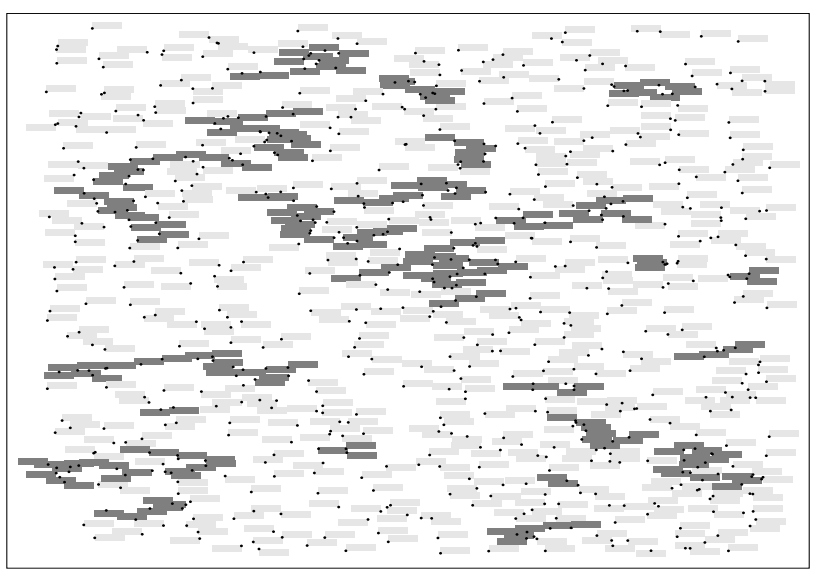

Hirsch’s Algorithm (222)

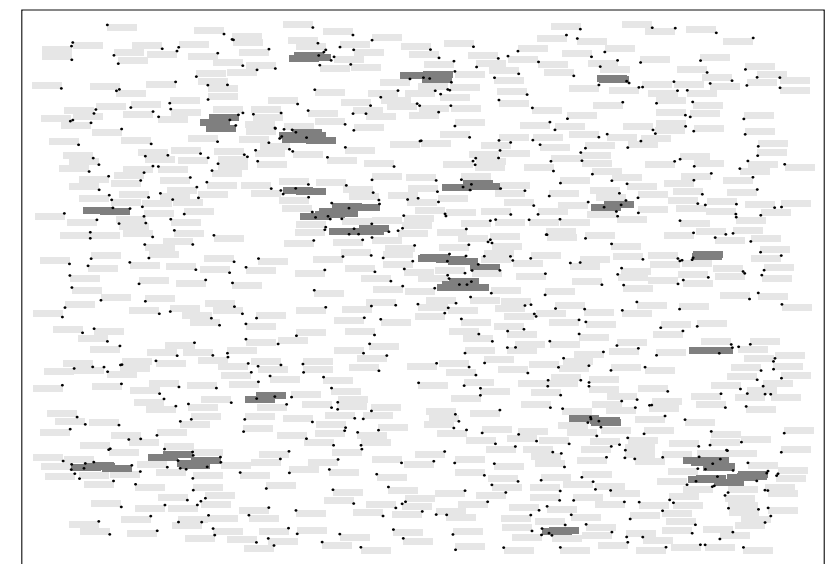

Simulated Annealing (75)

Figure 10: A sample map of 750 point features with labels placed by the six different algorithms. Labels printed in dark grey overplot other labels or points. Labels printed in light gray are free of overplots. Numbers in parenthesis indicate the final value of the objective function computed as the number of labels with overplots. 

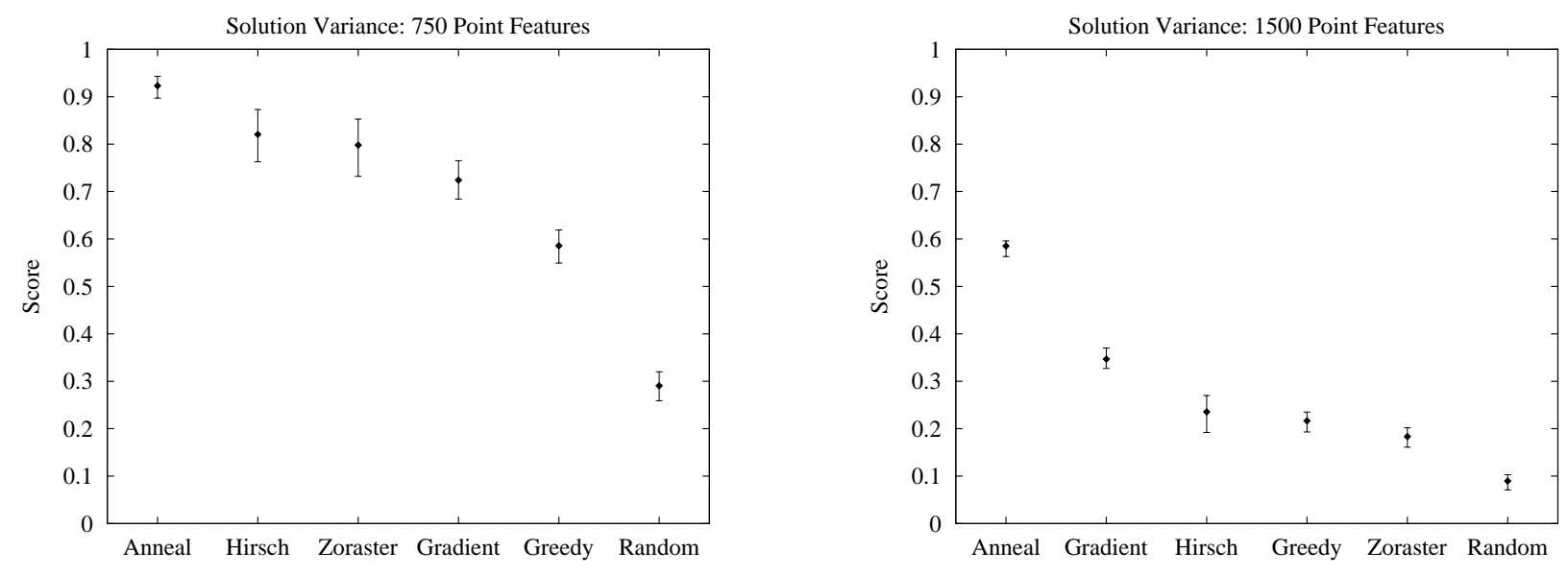

Figure 11: Range of results generated for 25 different labeling problems involving 750 and 1500 point features.
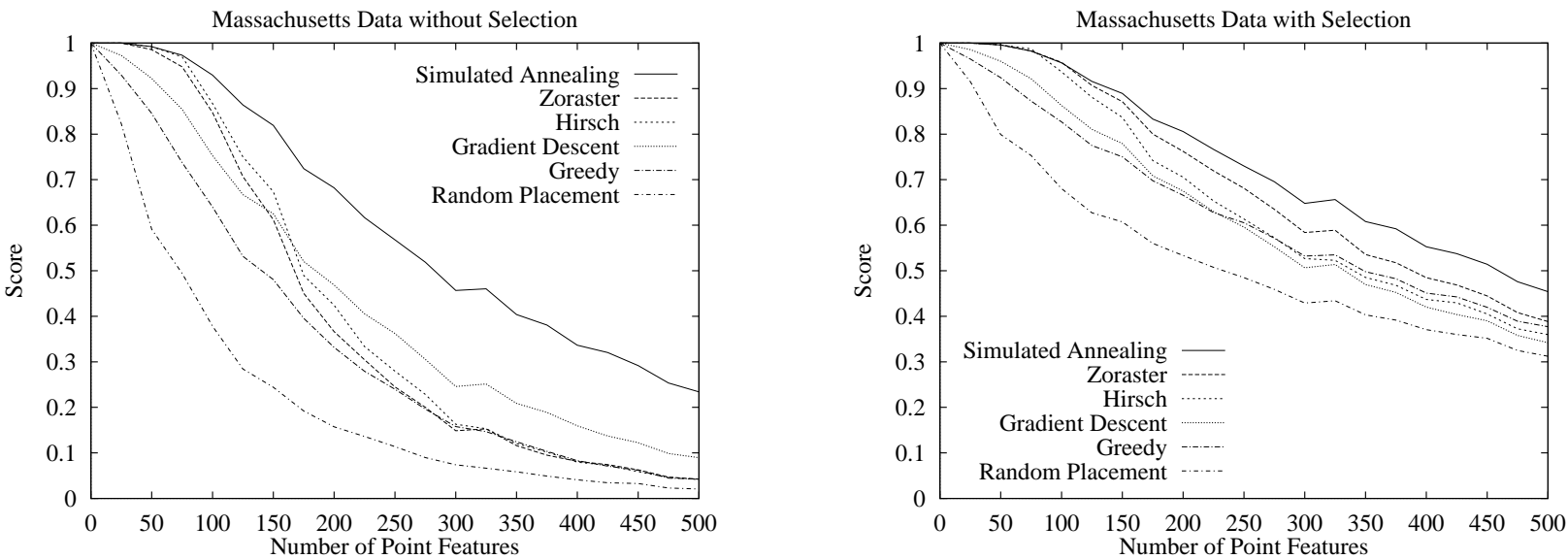

Figure 12: Results of empirical testing of six PFLP algorithms on GNIS data for Massachusetts with point selection prohibited and allowed.
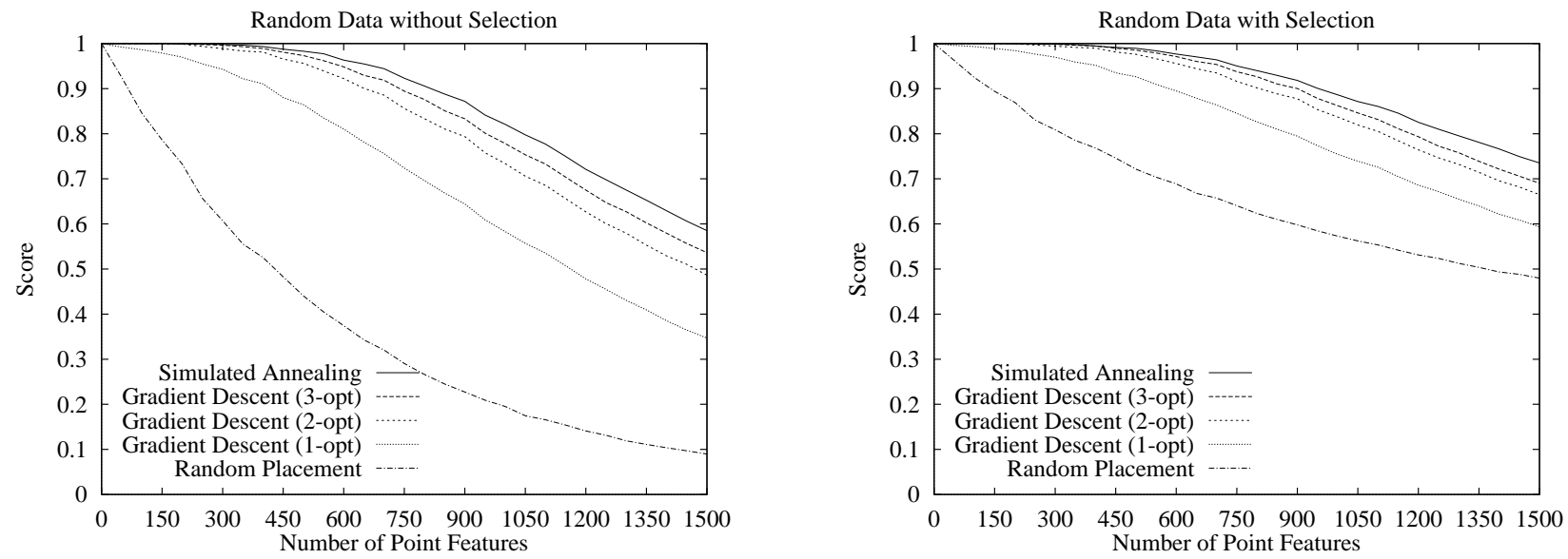

Figure 13: Results of empirical testing of discrete gradient-descent algorithms on randomly generated map data. 

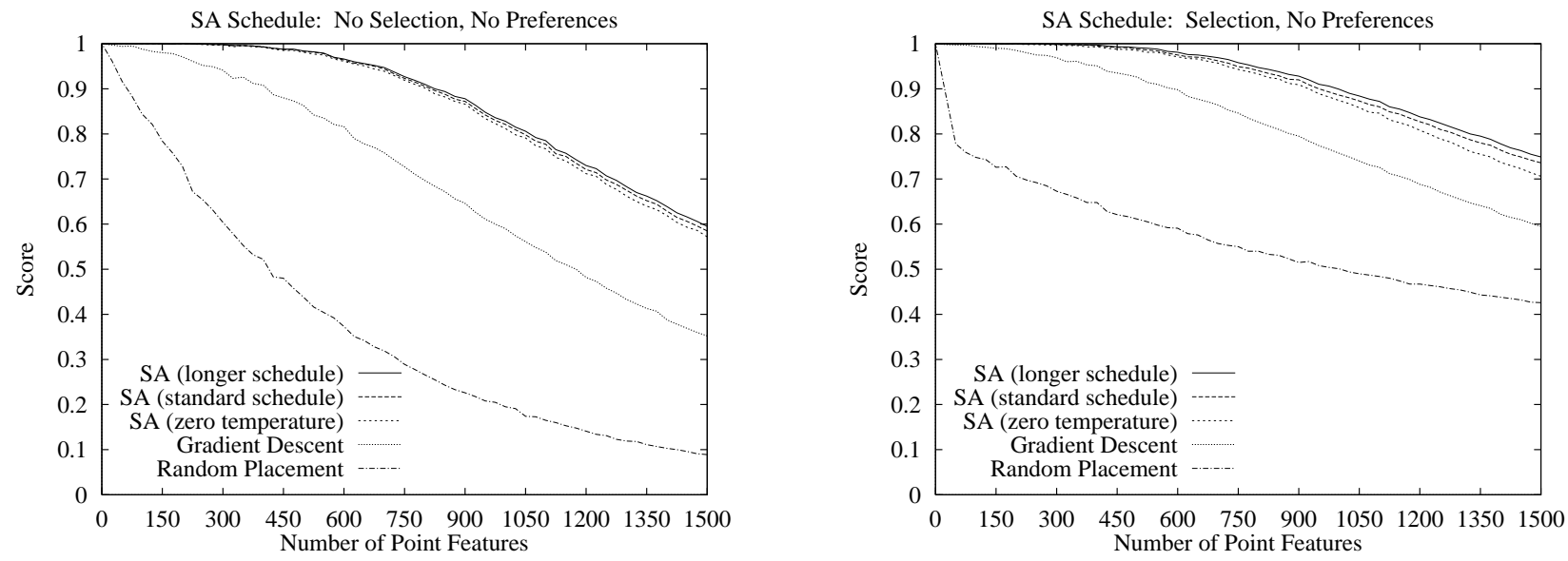

Figure 14: Comparison of annealing schedules against a gradient-descent algorithm without cartographic preferences.
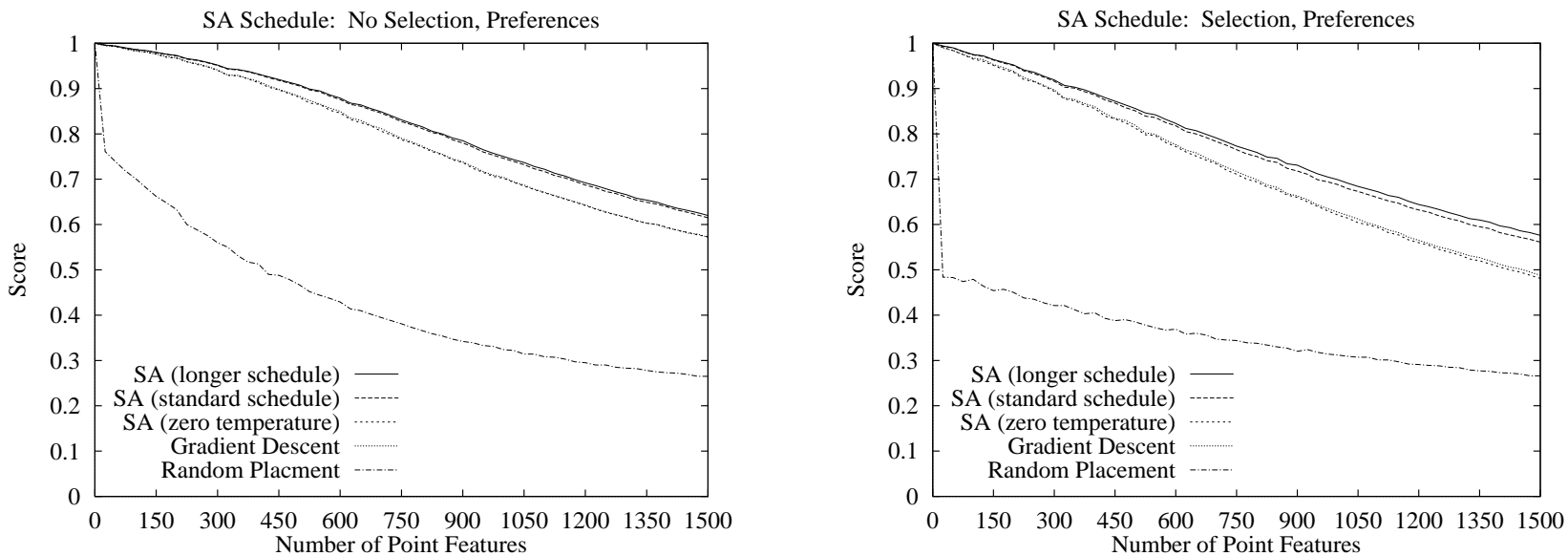

Figure 15: Comparison of annealing schedules against a gradient-descent algorithm with cartographic preferences. (Note that the lines corresponding to zero-temperature annealing and gradient descent lie very close together).
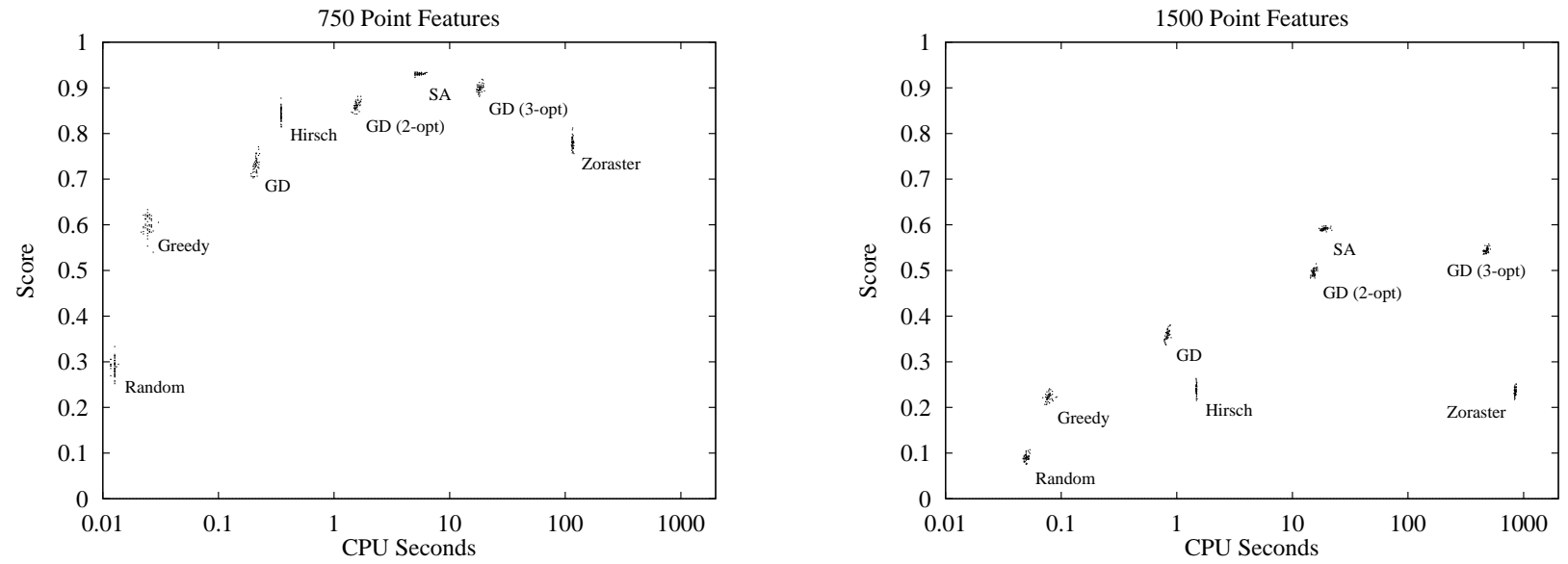

Figure 16: Running times for 50 different trials of 750 point features and 1500 point features. Note the logarithmic scale of the horizontal axis. 


\begin{tabular}{|l|c|}
\hline \multicolumn{1}{|c|}{ Algorithm } & Lines of C code \\
\hline \hline Random Placement & 20 \\
\hline Greedy & 79 \\
\hline Gradient Descent (1-opt) & 210 \\
\hline Simulated Annealing & 239 \\
\hline Zoraster & 346 \\
\hline Hirsch & 381 \\
\hline Gradient Descent (2-opt) & 1807 \\
\hline Gradient Descent (3-opt) & 2284 \\
\hline
\end{tabular}

Table 1: Lines of source code for label-placement algorithms

Unlike much of the previous work on label placement, the approach we have suggested cleanly separates the combinatorial-optimization aspect of the problem from the candidate-position modeling aspect. This way of stating the problem allows for the search algorithms discussed here to be used with more advanced cartographic positioning models. Modifying the algorithm to generate new sets of potential label positions, which is necessary to permit the labeling of line and area features, is accomplished easily, provided adequate models of line-feature (Ebinger and Goulette, 1990) and area-feature labeling (Carstensen, 1987; van Roessel, 1989) are available. Figure 17 shows a sample map involving all three feature types, as labeled by the simulated-annealing algorithm (Edmondson et al., 1995). Changing the objective function to allow for a priori placement preferences, sophisticated point selection, and complex interactions between labels and map symbology is also possible.

\section{Acknowledgments}

Much of this work was done while the second author was at Digital Equipment Corporation, Cambridge Research Laboratory. The research reported in this paper was funded in part by a contract with U S WEST Advanced Technologies, by Grants IRI-9157996 and 9350192 from the National Science Foundation, and by a grant from Digital Equipment Corporation. Andy Breeding, an information analyst at Digital Equipment Corporation, assisted us in the compilation of the bibliography. The authors wish to express their particular appreciation to the fourth anonymous reviewer for a very careful reading and many helpful suggestions, to Shawn Edmondson for his work on candidate-position algorithms for linear and area features, and to Tom Ngo for additional support. 


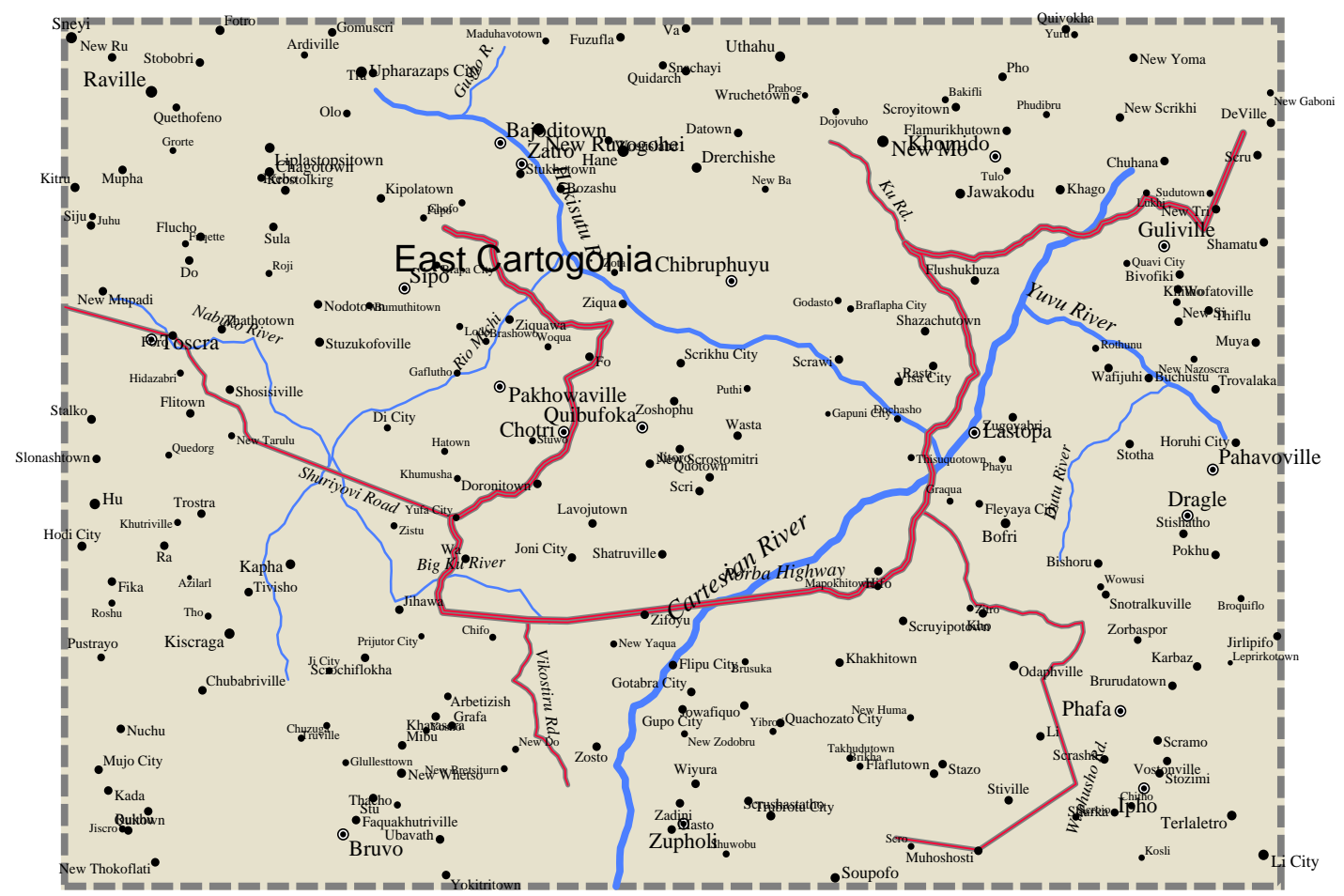

(a)

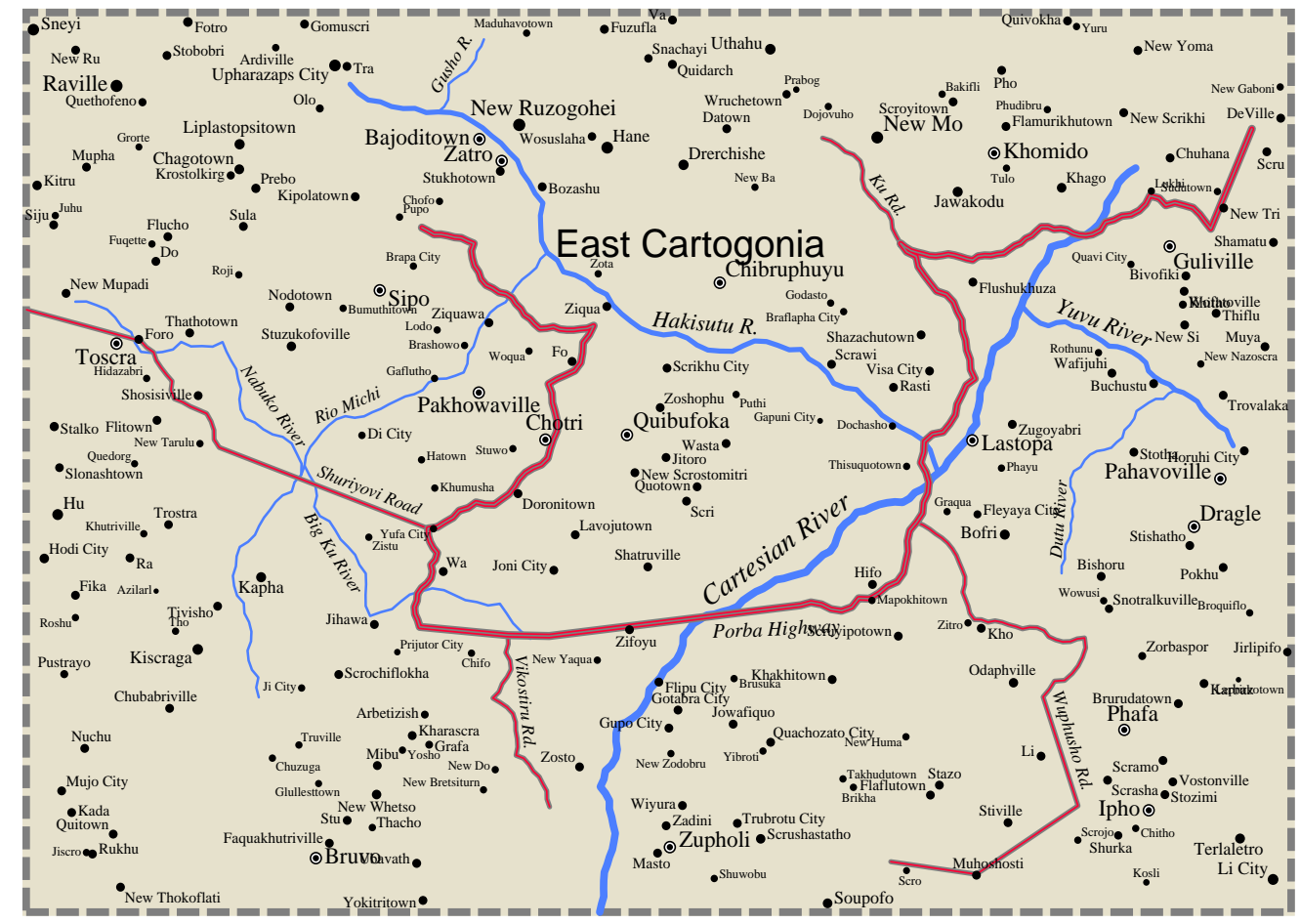

(b)

Figure 17: A map involving line, area, and point features labeled by the simulated-annealing algorithm. A random labeling is shown in (a). An intermediate configuration of the algorithm is shown in (b). The final labeling is shown in (c). The simulated-annealing algorithm converges from a random labeling to a final labeling in less than a second on a DEC 3000/400 AXP workstation. 


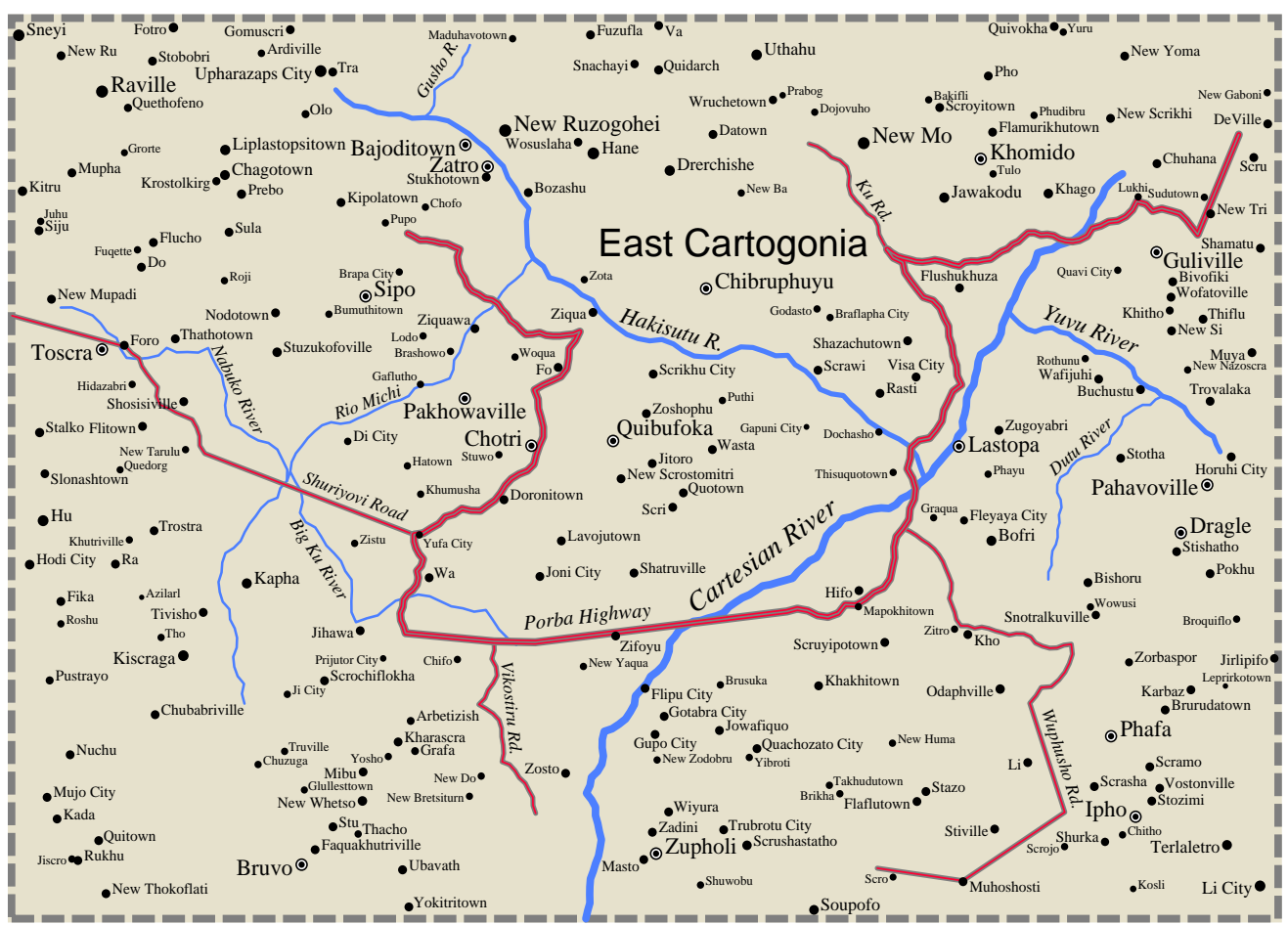

(c)

Figure 17: A map involving line, area, and point features labeled by the simulated-annealing algorithm. A random labeling is shown in (a). An intermediate configuration of the algorithm is shown in (b). The final labeling is shown in (c). The simulated-annealing algorithm converges from a random labeling to a final labeling in less than a second on a DEC 3000/400 AXP workstation. 


\section{References}

Ahn, J. and H. Freeman. 1984. A program for automatic name placement. Cartographica, 21(2\&3):101-109, Summer \& Autumn. Originally published in Proceedings of the Sixth International Symposium on Automated Cartography (AutoCarto Six), Ottawa/Hull, October 1983.

Carstensen, L. W. 1987. A comparison of simple mathematical approaches to the placement of spot symbols. Cartographica,24(3):46-63.

Cerny, V. 1985. A thermodynamical approach to the travelling salesman problem: An efficient simulation algorithm. Journal of Optimization Theory and Applications, 45:41-51.

Christensen, J., J. Marks, and S. Shieber. 1992. Labeling point features on maps and diagrams. Center for Research in Computing Technology, Harvard University, TR-25-92, December.

Christensen, J., J. Marks, and S. Shieber. 1993. Algorithms for cartographic label placement. Proceedings of the American Congress on Surveying and Mapping '93, February.

Christensen, J., J. Marks, and S. Shieber. 1994. Placing text labels on maps and diagrams. Graphics Gems IV. Academic Press, pages 497-504.

Cook, A. C. and C. B. Jones. 1990. A Prolog rule-based system for cartographic name placement. Computer Graphics Forum, 9:109-126.

Consorti, V., L.P. Cordella, and M. Iaccarino. 1993. Automatic lettering of cadastral maps. Proceedings of the International Conference on Document Analysis and Recognition, page 129-132, Tsukuba Science City, Japan, October.

Cromley, R. G. 1986. A spatial allocation analysis of the point annotation problem. In Proceedings of the Second International Symposium on Spatial Data Handling, pages 38-49, Seattle, Washington, July. International Geographical Union and International Cartographic Association.

Doerschler, J. S. and H. Freeman. 1992. A rule-based system for dense-map name placement. Communications of the Association of Computing Machinery, 35(1):68-79, January.

Ebinger, L. R. and A. M. Goulette. 1990. Noninteractive automated names placement for the 1990 decennial census. Cartography and Geographic Information Systems, 17(1):69-78, January.

Edmondson, S., J. Christensen, J. Marks, and S. Shieber. 1995. A general cartographic labeling algorithm. In preparation.

Feigenbaum, M. 1994. Method and apparatus for automatically generating symbol images against a background image without collision utilizing distance-dependent attractive and repulsive forces in a computer simulation. Assigned to Hammond Inc., Maplewood, New Jersey. U.S. Patent \#5,355,314. Patent filed 11/5/93, received 10/11/94.

Fisher, M. L. 1981. The Lagrangian relaxation method for solving integer programming problems. Management Science, 27:118.

Formann, M. and F. Wagner. 1991. A packing problem with applications to lettering of maps. In Proceedings of the Seventh Annual Symposium on Computational Geometry, pages 281-288, North Conway, New Hampshire, July. ACM.

Freeman, H. and J. Ahn. 1987. On the problem of placing names in a geographic map. International Journal of Pattern Recognition and Artificial Intelligence, 1(1):121-140.

Freeman, H. 1988. An Expert System for the automatic placement of names on a geographical map. Information Sciences, 45:367-378. 
Hirsch, S. A. 1982. An algorithm for automatic name placement around point data. The American Cartographer, 9(1):5-17.

Holland, J. H. 1975. Adaptation in Natural and Artificial Systems. University of Michigan Press, Ann Arbor.

Imhof, E. 1962. Die Anordnung der Namen in der Karte. International Yearbook of Cartography, 2:93-129.

Imhof, E. 1975. Positioning names on maps. The American Cartographer, 2(2):128-144.

Johnson, D. S. 1990. Local optimization and the traveling salesman problem. In Proceedings of the 17th Colloqium on Automata, Languages, and Programming, pages 446-461. Springer-Verlag.

Johnson, D. S., C. R. Aragon, L. A. McGeoch, and C. Schevon. 1989. Optimization by simulated annealing: An experimental evaluation; part I, graph partitioning. Operations Research, 37(6):865-892.

Johnson, D. S., C. R. Aragon, L. A. McGeoch, and C. Schevon. 1991. Optimization by simulated annealing: An experimental evaluation; part II, graph coloring and number partitioning. Operations Research, 39(3):378-406.

Jones, C. 1989. Cartographic name placement with Prolog. IEEE Computer Graphics and Applications, 9(5):36-47, September.

Kato, T. and H. Imai. 1988. The NP-completeness of the character placement problem of 2 or 3 degrees of freedom. Record of Joint Conference of Electrical and Electronic Engineers in Kyushu, 1138. In Japanese.

Kirkpatrick, S., C. D. Gelatt Jr., and M. P. Vecchi. 1983. Optimization by simulated annealing. Science, 220:671-680.

Korf, R. E. 1988. Search: A survey of recent results. In H. E. Shrobe, editor, Exploring Artificial Intelligence: Survey Talks from the National Conferences on Artificial Intelligence. Morgan Kaufmann, San Mateo, California, pages 197-237.

Langran, G. E. and T. K. Poiker. 1986. Integration of name selection and name placement. In Proceedings of the Second International Symposium on Spatial Data Handling, pages 50-64, Seattle, Washington, July. International Geographical Union and International Cartographic Association.

Marks, J. and S. Shieber. 1991. The computational complexity of cartographic label placement. Technical Report TR-05-91, Harvard University, March.

Mower, J. E. 1986. Name placement of point features through constraint propagation. In Proceedings of the Second International Symposium on Spatial Data Handling, pages 65-73, Seattle, Washington, July. International Geographical Union and International Cartographic Association.

Mower, J. E. 1993. Automated feature and name placement on parallell computers. In Cartography and Geographic Information Systems, 20(2):69-82.

Noma, E. 1987. Heuristic method for label placement in scatterplots. Psychometrika, 52(3):463-468.

Sahni, S. 1974. Computationally related problems. SIAM Journal on Computing, 3:262-279.

United States Geological Survey, National Mapping Division. 1990. Geographic Names Information System, November.

van Roessel, J. W. 1989. An algorithm for locating candidate labeling boxes within a polygon. The American Cartographer, 16(3):201-209.

Wu, C. V. and B. P. Buttenfield. 1991. Reconsidering rules for point-feature name placement. Cartographica, 28(1):10-27, Spring. 
Yoeli, P. 1972. The logic of automated map lettering. The Cartographic Journal, 9(2):99-108, December.

Zoraster, S. 1986. Integer programming applied to the map label placement problem. Cartographica, 23(3):16-27.

Zoraster, S. 1990. The solution of large 0-1 integer programming problems encountered in automated cartography. Operations Research, 38(5):752-759, September-October.

Zoraster, S. 1991. Expert systems and the map label placement problem. Cartographica, 28(1):1-9, Spring. 